\title{
Stochastic Stability of a Fractional Viscoelastic Plate Driven by Non-Gaussian Colored Noise
}

\section{Dongliang Hu}

College of Mechanics and Materials, Hohai University

Yong Huang ( $\nabla$ yhuang1984@163.com )

School of Energy and Power Engineering, Nanjing University of Science and Technology

\section{Research Article}

Keywords: Stochastic stability, Moment Lyapunov exponent, Largest Lyapunov exponent, Perturbation method, Monte Carlo simulation, Fractional order calculus

Posted Date: September 13th, 2021

DOI: https://doi.org/10.21203/rs.3.rs-844087/v1

License: (1) This work is licensed under a Creative Commons Attribution 4.0 International License. Read Full License

Version of Record: A version of this preprint was published at Nonlinear Dynamics on February 13th, 2022. See the published version at https://doi.org/10.1007/s11071-022-07278-w. 


\title{
Stochastic stability of a fractional viscoelastic plate driven by non-Gaussian colored noise
}

\author{
Dongliang $\mathrm{Hu}^{\text {a }}$ Yong Huang ${ }^{\mathrm{b}^{*}}$ \\ ${ }^{a}$ College of Mechanics and Materials, Hohai University, Nanjing 211100, China \\ ${ }^{b}$ School of Energy and Power Engineering, Nanjing University of Science and Technology, \\ Nanjing, 210094, China \\ *Author for correspondence \\ E-mail: dlhu@hhu.edu.cn (Dongliang Hu)
}

yhuang1984@163.com (Yong Huang)

\begin{abstract}
In this paper, the moment Lyapunov exponent and stochastic stability of a fractional viscoelastic plate driven by non-Gaussian colored noise is investigated. Firstly, the stochastic dynamic equations with two degrees of freedom are established by piston theory and Galerkin approximate method. The fractional Kelvin-Voigt constitutive relation is used to describe the material properties of the viscoelastic plate, which leads to that the fractional derivation term is introduced into the stochastic dynamic equations. And the noise is simplified into an Ornstein-Uhlenbeck process by utilizing the path-integral method. Then, via the singular perturbation method, the approximate expansions of the moment Lyapunov exponent are obtained, which agree well with the results obtained by the Monte Carlo simulations. Finally, the effects of the noise, viscoelastic parameters and system parameters on the stochastic dynamics of the viscoelastic plate are discussed.
\end{abstract}

Keywords Stochastic stability; Moment Lyapunov exponent; Largest Lyapunov exponent; Perturbation method; Monte Carlo simulation; Fractional order calculus

\section{Introduction}

In the past several decades, the flutter problem of plates has attracted more and more attention with the development of supersonic aircraft. Flutter instability will lead to the decrease of structure life and even cause structural failure. Viscoelastic materials are widely used in various parts of aircraft and high-speed trains because of their excellent vibration damping properties, which can suppress the fluttering of plates. On the other hand, random factors exist in nature, such as high winds, ocean waves, earthquakes, atmospheric turbulence, and so on. Therefore, determining the dynamic behaviors of the viscoelastic systems under random excitation is of great help for engineering applications, which also has been investigated by many researchers [1-5].

Although plenty of achievements have been made in the study of stochastic dynamics of viscoelastic systems [6-8], the research on stochastic flutter of the viscoelastic plates is still limited. Potapov[9] investigated the stochastic flutter of a viscoelastic plate under white noise 
excitation through the stability conditions in the first and second statistical moments. Based on the results of largest Lyapunov exponent determined by the stochastic averaging method, Ariaratnam and Abdelrahman[10] examined the almost sure stability of a viscoelastic plate in the supersonic gas flow. Later, by applying numerical evaluation of the largest Lyapunov exponent, Potapov[11] studied the stability of elastic and viscoelastic plates under a stochastic excitation. The largest Lyapunov exponent also has been used to determine the almost sure stability of other viscoelastic systems, such as viscoelastic beam[12], viscoelastic column[13], non-gyroscopic viscoelastic system[14], and so on.

According to the large deviation theory[15], even for the stochastic dynamical system with almost sure stable, the $p$ th moment of response can be unstable and grow exponentially. Hence, it is necessary to investigate the $p$ th moment Lyapunov exponent of a stochastic dynamical system. The $p$ th moment Lyapunov exponent is defined as follows:

$$
\Lambda\left(p, \mathbf{x}_{0}\right)=\lim _{t \rightarrow+\infty} \frac{1}{t} \log E\left[\left\|\mathbf{x}\left(t ; \mathbf{x}_{0}\right)\right\|^{p}\right],
$$

where $\mathbf{x}\left(t ; \mathbf{x}_{0}\right)$ is a solution process to a stochastic dynamical system. If $\Lambda\left(p, \mathbf{x}_{0}\right)<0$, then $E\left[\left\|\mathbf{x}\left(t ; \mathbf{x}_{0}\right)\right\|^{p}\right] \rightarrow 0$ as $t \rightarrow+\infty$, which implies that the $p$ th moment is stable; otherwise, it is unstable. In Ref.[16], it has been disclosed that the limit of Eq. (1) exists and is independent of $\mathbf{x}_{0}$ under the specified conditions. Thus $\Lambda\left(p, \mathbf{x}_{0}\right)$ can be expressed as $\Lambda(p)$, which is a convex analytic function in $p \in \mathbf{R}$. And the largest Lyapunov exponent $\lambda$ is just the first-order term coefficient of moment Lyapunov exponent with respect to $p$ [17], i.e.

$$
\lambda=\left.\frac{\mathrm{d}}{\mathrm{d} p} \Lambda(p)\right|_{p=0}=\lim _{t \rightarrow \infty} \frac{1}{t} \log \left\|\mathbf{x}\left(t, \mathbf{x}_{0}\right)\right\| .
$$

Based on the pth moment Lyapunov exponent, the stochastic stability, D-bifurcation and P-bifurcation $[15,18]$ of a random system can be determined. Therefore, the $p$ th moment Lyapunov exponent can describe the stochastic dynamic behavior of a system more profoundly and completely.

Although the $p$ th moment Lyapunov exponent is very significant in the research of stochastic dynamics of a system, it is especially difficult to calculate the moment Lyapunov exponent. In recent years, researchers have studied the moment Lyapunov exponent for different stochastic dynamical systems, and achieved gratifying results[19-24]. However, the research on the viscoelastic plates is still limited. For a wide-band noise or non-Gaussian colored noise excited viscoelastic plate, Huang[25, 26] studied the $p$ th moment Lyapunov exponent and obtained an asymptotic expansion of the moment Lyapunov exponent using the stochastic averaging method. By determining the moment Lyapunov exponent, Deng[27] 
examined the stochastic stability of a viscoelastic plate under bounded noise excitation. Recently, $\mathrm{Wu}$ [28] discussed the moment stability of a viscoelastic plate under non-Gaussian colored noise excitation by calculating the $p$ th moment Lyapunov exponent.

With the development of fractional calculus theory, it is found that the integer-order viscoelastic model cannot meet the actual needs, while the fractional viscoelastic model is more accurate and more in line with practical engineering. The constitutive relation of viscoelastic materials can be described by the fractional Kelvin-Voigt model, which also has been adopted in a lot of researches on viscoelastic systems [29-31]. For a fractional viscoelastic plate excited by non-Gaussian colored noise, the stochastic stability and $p$ th moment Lyapunov exponent are investigated in the present paper. The fractional KelvinVoigt model is introduced to describe the material properties of the viscoelastic plates. And via the path-integral method[32-34], the non-Gaussian colored noise is reduced to an Ornstein-Uhlenbeck $(\mathrm{O}-\mathrm{U})$ process. By applying the singular perturbation method and Fourier series expansion, the approximate analytical results of the $p$ th moment Lyapunov exponent and largest Lyapunov exponent are given. Furthermore, the numerical results of the original system are also obtained by using Monte Carle simulation. Finally, based on the $p$ th moment Lyapunov exponent and largest Lyapunov exponent, the impacts of the noise, viscoelastic parameters and system parameters on the stochastic dynamics of the fractional viscoelastic plate are studied and discussed in detail.

\section{Formulation}

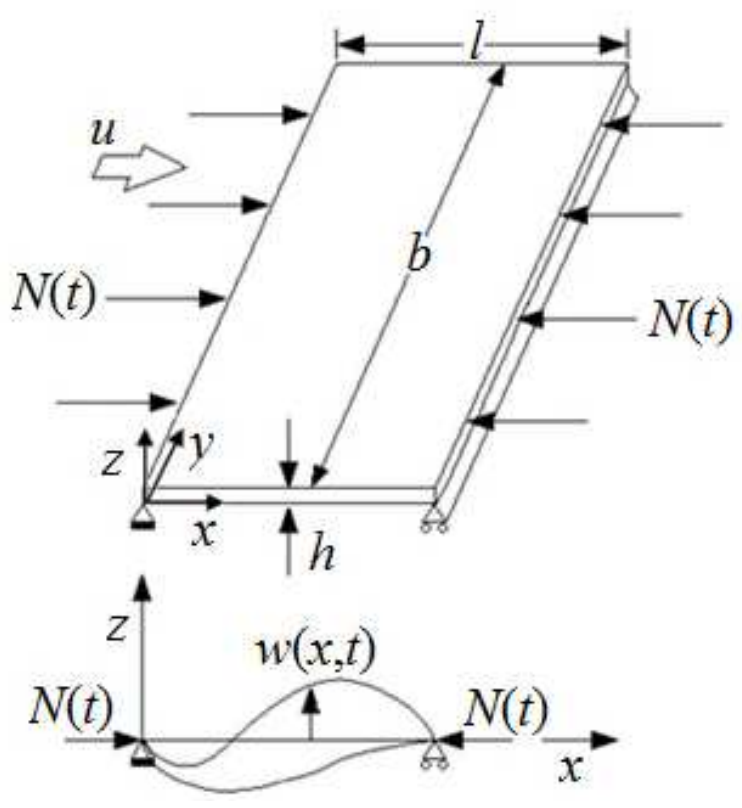

Fig.1 Model of the plate 
Considered an infinitely long viscoelastic plate with simply supported conditions is shown in Figure 1. Based on the classical bending theory of the small deflection of thin plates, the governing equation for the viscoelastic plate with small oscillation is given as follows

$$
\rho h \frac{\partial^{2} w(x, t)}{\partial t^{2}}+\rho h c \frac{\partial w(x, t)}{\partial t}+N(t) \frac{\partial^{2} w(x, t)}{\partial x^{2}}-\frac{\partial^{2} M_{x}}{\partial x^{2}}+P(x, t)=0,
$$

where

$$
M_{x}=\int_{-h / 2}^{h / 2} \sigma_{x} z d z, \quad \varepsilon_{x}=-\frac{\partial^{2} w(x, t)}{\partial x^{2}} z
$$

$w(x, y, t)$ is the transversal displacement of plate; $M_{x}$ is the internal moment per unit length; $\sigma_{x}$, $\varepsilon_{x}$ are the stress and strain of the plate. $b, l, h$ are the length, width and thickness of plate, respectively, and $b \rightarrow \infty . \rho$ is the material density; $c$ is the viscous damping coefficient per unit length; $N(t)$ is the external random force in-plane load per unit length. $P(x, t)$ is aerodynamic force per unit area, which can be obtained by applying the piston theory.

$$
P(x, t)=\chi \frac{P_{\infty}}{C_{\infty}}\left[\frac{\partial w(x, t)}{\partial t}+u \frac{\partial w(x, t)}{\partial x}\right],
$$

where $u, \chi, C_{\infty}$, and $P_{\infty}$ represent the flow velocity, gas flow polytropy index, undisturbed gas speed, and undisturbed gas pressure, respectively.

The constitutive relation of viscoelastic materials can be given as

$$
\sigma(t)=\left[E+\gamma_{0} \cdot D_{t}^{\alpha}\right] \varepsilon(t)
$$

where $E$ is the elastic modulus; $\gamma_{0}$ is the viscoelastic coefficient; $D_{t}^{\alpha}$ represents the Riemann-Liouville fractional derivative with $\alpha \in(0,1)$, which is defined as

$$
D_{t}^{\alpha} f(t)=\frac{1}{\Gamma(1-\alpha)} \frac{d}{d t} \int_{0}^{t}(t-\tau)^{-\alpha} f(\tau) d \tau .
$$

Substituting Eqs.(4-6) into Eq. (3), we can obtain

$$
\begin{array}{r}
\rho h \frac{\partial^{2} w(x, t)}{\partial t^{2}}+\rho h c \frac{\partial w(x, t)}{\partial t}+N(t) \frac{\partial^{2} w(x, t)}{\partial x^{2}}+E I \frac{\partial^{4} w(x, t)}{\partial x^{4}} \\
+\gamma_{0} I \cdot D_{t}^{\alpha} \frac{\partial^{4} w(x, t)}{\partial x^{4}}+\chi \frac{P_{\infty}}{C_{\infty}}\left[\frac{\partial w(x, t)}{\partial t}+u \frac{\partial w(x, t)}{\partial x}\right]=0 .
\end{array}
$$

Bolotin[35] pointed out that quantitative results are reliably predicted with the use of the first two modes. Therefore, the transversal displacement $w(x, t)$ can be expressed as

$$
w(x, t)=h_{1}(t) \sin \frac{\pi x}{l}+h_{2}(t) \sin \frac{2 \pi x}{l},
$$

which satisfies the boundary conditions: 


$$
\begin{aligned}
& w(0, t)=w(l, t)=0, \\
& \left.\frac{\partial^{2} w(x, t)}{\partial x^{2}}\right|_{x=0}=\left.\frac{\partial^{2} w(x, t)}{\partial x^{2}}\right|_{x=l}=0 .
\end{aligned}
$$

From Eq.(7) and Eq.(8), Eq.(9) can be obtained by using the Galerkin method.

$$
\begin{aligned}
& l_{1}+\frac{C}{\rho h} h_{1}^{\&}+\frac{E I}{\rho h}\left(\frac{\pi}{l}\right)^{4}(1-\xi(t)) h_{1}+\frac{\gamma_{0} I}{\rho h}\left(\frac{\pi}{l}\right)^{4} \cdot D_{t}^{\alpha} h_{1}-\frac{8 \chi u P_{\infty}}{3 l \rho h C_{\infty}} h_{2}=0, \\
& { }_{2}+\frac{C}{\rho h} l_{2}^{\&}+\frac{E I}{\rho h}\left(\frac{\pi}{l}\right)^{4}(16-4 \xi(t)) h_{2}+\frac{\gamma_{0} I}{\rho h}\left(\frac{2 \pi}{l}\right)^{4} \cdot D_{t}^{\alpha} h_{2}+\frac{8 \chi u P_{\infty}}{3 l \rho h C_{\infty}} h_{1}=0,
\end{aligned}
$$

where

$$
C=\rho h c+\frac{\chi P_{\infty}}{C_{\infty}}, \quad \xi(t)=\frac{l^{2} N(t)}{\pi^{2} E I}
$$

Let $t^{\prime}=c_{1} t$ and via a series of transformations and simplifications, the system (9) becomes, by introducing small parameter $\varepsilon \quad(0<\varepsilon<<1)$,

$$
\begin{aligned}
& +2 \varepsilon^{2} \beta \&+\omega_{1}^{2} q_{1}+\varepsilon^{2} \gamma c_{1}^{\alpha} \omega_{1}\left(b_{11} \cdot D_{t^{\prime}}^{\alpha} q_{1}+b_{12} \cdot D_{t^{\prime}}^{\alpha} q_{2}\right)+\varepsilon \omega_{1}\left(k_{11} q_{1}+k_{12} q_{2}\right) \xi\left(t^{\prime}\right)=0, \\
& +2 \varepsilon^{2} \beta \&+\omega_{2}^{2} q_{2}+\varepsilon^{2} \gamma c_{1}^{\alpha} \omega_{2}\left(b_{21} \cdot D_{t^{\prime}}^{\alpha} q_{1}+b_{22} \cdot D_{t^{\prime}}^{\alpha} q_{2}\right)+\varepsilon \omega_{2}\left(k_{21} q_{1}+k_{22} q_{2}\right) \xi\left(t^{\prime}\right)=0,
\end{aligned}
$$

where assuming the damping term and viscoelastic term are both the second-order small quantity of parameter $\varepsilon$, and the random term is a small quantity of parameter $\varepsilon$.

$$
\begin{aligned}
& {\left[\begin{array}{l}
h_{1} \\
h_{2}
\end{array}\right]=\mathbf{T}\left[\begin{array}{l}
q_{1} \\
q_{2}
\end{array}\right], \quad \mathbf{T}=\left[\begin{array}{cc}
-a & -a c^{*} \\
\omega_{1}^{2}-1 & c^{*}\left(\omega_{2}^{2}-1\right)
\end{array}\right],} \\
& b_{11}=\frac{-225+17 a_{0}}{2 a_{0} \omega_{1}}, \quad b_{12}=\frac{\left(-225-15 a_{0}\right) c^{*}}{2 a_{0} \omega_{1}}, \\
& b_{21}=\frac{225-15 a_{0}}{2 a_{0} \omega_{2} c^{*}}, \quad b_{22}=\frac{225+17 a_{0}}{2 a_{0} \omega_{2}} \\
& k_{11}=\frac{45-5 a_{0}}{2 a_{0} \omega_{1}}, \quad k_{12}=\frac{\left(45+3 a_{0}\right) c^{*}}{2 a_{0} \omega_{1}}, \\
& k_{21}=\frac{-45+3 a_{0}}{2 a_{0} \omega_{2} c^{*}}, \quad k_{22}=\frac{-45-5 a_{0}}{2 a_{0} \omega_{2}}, \\
& \omega_{1,2}^{2}=\frac{17}{2} \mathrm{~m} \frac{a_{0}}{2}, \quad a_{0}=\sqrt{225-4 a^{2}}, \quad a=\frac{8 u \chi P_{\infty} l^{3}}{3 C_{\infty} \pi^{4} E I}, \\
& \beta=\frac{C l^{2}}{2 \sqrt{\rho h E I}}, \quad \gamma=\frac{\gamma_{0}}{E}, \quad c_{1}=\frac{\pi^{2}}{l^{2}} \sqrt{\frac{E I}{\rho h}}, \quad c^{*} \text { is a constant. }
\end{aligned}
$$




\section{Non-Gaussian colored noise case}

Suppose that the process $\xi\left(t^{\prime}\right)$ in system (10) is a non-Gaussian colored noise given as follows

$$
\begin{gathered}
\frac{\mathrm{d} \xi\left(t^{\prime}\right)}{\mathrm{d} t^{\prime}}=-\frac{1}{\tau} \frac{\mathrm{d}}{\mathrm{d} \xi} V_{r}(\xi)+\frac{1}{\tau} \eta\left(t^{\prime}\right), \\
V_{r}(\xi)=\frac{D}{\tau(r-1)} \ln \left[1+\frac{\tau}{D}(r-1) \frac{\xi^{2}}{2}\right],
\end{gathered}
$$

where $\tau, D$ are the noise correlation time and noise intensity, respectively. $r$ is the departure coefficient, denoting the departure from the noise. $\eta\left(t^{\prime}\right)$ is a Gaussian white noise and its statistical properties are

$$
\left\langle\eta\left(t^{\prime}\right)=0\right\rangle,\left\langle\eta\left(t^{\prime}\right) \eta(s)\right\rangle=2 D \delta\left(t^{\prime}-s\right)
$$

For $r \rightarrow 1$, the process $\xi\left(t^{\prime}\right)$ is an O-U process with correlation time $\tau$, and its correlation function is expressed as $\left\langle\xi\left(t^{\prime}\right) \xi(s)\right\rangle=(D / \tau) e^{-\left|t^{\prime}-s\right| / \tau}$. And $\xi\left(t^{\prime}\right)$ is further simplified to a Gaussian white noise as $\tau \rightarrow 0$. As in Ref.[36], the stationary probability density $P_{s}(\xi)$ of Eq. (11) can be normalized if and only if $r \in(-\infty, 3)$, which is given as

$$
P_{s}(\xi)=\frac{1}{Z}\left[1+\frac{\tau}{D}(r-1) \frac{\xi^{2}}{2}\right]^{-1 / r-1}
$$

where $Z$ is a normalization constant. According to Eq. (14), one can easily obtain the statistical properties of the process $\xi\left(t^{\prime}\right)$ :

$$
\begin{aligned}
& \left\langle\xi\left(t^{\prime}\right)\right\rangle=0, \\
& \left\langle\xi^{2}\left(t^{\prime}\right)\right\rangle= \begin{cases}2 D /[\tau(5-3 r)], & r \in(-\infty, 5 / 3) \\
\infty, & r \in(5 / 3,3)\end{cases}
\end{aligned}
$$

As $|r-1|<<1$, utilizing a path-integral method[36, 37], one may get the following expression,

$$
\frac{1}{\tau} \frac{\mathrm{d}}{\mathrm{d} \xi} V_{r}(\xi)=\frac{\xi}{\tau}\left[1+\frac{\tau}{D}(r-1) \frac{\xi^{2}}{2}\right]^{-1} \approx \frac{\xi}{\tau}\left[1+\frac{\tau}{D}(r-1) \frac{\left\langle\xi^{2}\right\rangle}{2}\right]^{-1}=\frac{\xi}{\tau_{1}}
$$

with the effective noise correlation time defined as 


$$
\tau_{1}=\frac{2(2-r)}{5-3 r} \tau,
$$

and the associated noise intensity defined as

$$
D_{1}=\left(\frac{2(2-r)}{5-3 r}\right)^{2} D
$$

Hence, Eq. (10) is reduced to an O-U process with the associated noise intensity $D_{1}$ and correlation time $\tau_{1}$,

$$
\frac{\mathrm{d} \xi\left(t^{\prime}\right)}{\mathrm{d} t^{\prime}}=-\frac{1}{\tau_{1}} \xi\left(t^{\prime}\right)+\frac{1}{\tau_{1}} \eta_{1}\left(t^{\prime}\right), \text { i.e., } \mathrm{d} \xi\left(t^{\prime}\right)=-\alpha_{0} \xi\left(t^{\prime}\right) \mathrm{d} t^{\prime}+\sigma_{0} \mathrm{od} W\left(t^{\prime}\right),
$$

where

$$
\left\{\begin{array}{lc}
\left\langle\eta_{1}\left(t^{\prime}\right)\right\rangle=0, & \left\langle\eta_{1}\left(t^{\prime}\right) \eta_{1}(s)\right\rangle=2 D_{1} \delta\left(t^{\prime}-s\right) \\
\alpha_{0}=1 / \tau_{1}, & \sigma_{0}=\sqrt{2 D_{1}} / \tau_{1}=\sqrt{2 D} / \tau
\end{array}\right.
$$

$W\left(t^{\prime}\right)$ is a wiener process with unit intensity, and the symbol "o" denotes the Stratonovich stochastic integral. Because of diffusion coefficient of the O-U process (19), the correction term of Eq. (19) is equal to zero. i.e., the Itô stochastic differential equation of Eq. (19) can be written as

$$
\mathrm{d} \xi\left(t^{\prime}\right)=-\alpha_{0} \xi\left(t^{\prime}\right) \mathrm{d} t^{\prime}+\sigma_{0} \mathrm{~d} W\left(t^{\prime}\right)
$$

with the power spectral density

$$
S(\omega)=\frac{\sigma_{0}^{2}}{\alpha_{0}^{2}+\omega^{2}}
$$

\section{Reduction of fractional differentiation}

Applying the following transformation:

$$
q_{i}=x_{2 i-1}, \underset{l}{\&}=\omega_{i} x_{2 i}, i=1,2
$$

Eq. (10) is represented as

$$
\begin{aligned}
& \mathbf{x}=\mathbf{A} \mathbf{x}+\varepsilon^{2} \mathbf{B} D_{t^{\prime}}^{\alpha} \mathbf{x}+\varepsilon \mathbf{K} \mathbf{x} \xi\left(t^{\prime}\right), \\
& \mathrm{d} \xi\left(t^{\prime}\right)=-\alpha_{0} \xi\left(t^{\prime}\right) \mathrm{d} t^{\prime}+\sigma_{0} \mathrm{~d} W\left(t^{\prime}\right), \quad \xi \in M
\end{aligned}
$$

where 
$\mathbf{A}=\left[\begin{array}{cccc}0 & \omega_{1} & 0 & 0 \\ -\omega_{1} & -2 \varepsilon^{2} \beta & 0 & 0 \\ 0 & 0 & 0 & \omega_{2} \\ 0 & 0 & -\omega_{2} & -2 \varepsilon^{2} \beta\end{array}\right], \mathbf{B}=-\gamma c_{1}^{\alpha}\left[\begin{array}{cccc}0 & 0 & 0 & 0 \\ b_{11} & 0 & b_{12} & 0 \\ 0 & 0 & 0 & 0 \\ b_{21} & 0 & b_{22} & 0\end{array}\right], \quad \mathbf{K}=\left[\begin{array}{cccc}0 & 0 & 0 & 0 \\ -k_{11} & 0 & -k_{12} & 0 \\ 0 & 0 & 0 & 0 \\ -k_{21} & 0 & -k_{22} & 0\end{array}\right]$

Based on the transformation

$$
\begin{aligned}
& x_{1}=e^{\rho} \cos \theta \cos \phi_{1}, x_{2}=-e^{\rho} \cos \theta \sin \phi_{1}, \\
& x_{3}=e^{\rho} \sin \theta \cos \phi_{2}, x_{4}=-e^{\rho} \sin \theta \sin \phi_{2},
\end{aligned}
$$

Eq. (24) can be written as

$$
\begin{aligned}
& \rho \&=\varepsilon^{2} \rho_{2}\left(\rho, \theta, \phi_{1}, \phi_{2}\right)+\varepsilon \rho_{1}\left(\theta, \phi_{1}, \phi_{2}\right) \xi\left(t^{\prime}\right), \\
& \theta^{\&}=\varepsilon^{2} s_{2}\left(\rho, \theta, \phi_{1}, \phi_{2}\right)+\varepsilon s_{1}\left(\theta, \phi_{1}, \phi_{2}\right) \xi\left(t^{\prime}\right), \\
& \phi_{1}^{\&}=\varepsilon^{2} h_{12}\left(\rho, \theta, \phi_{1}, \phi_{2}\right)+\varepsilon h_{11}\left(\theta, \phi_{1}, \phi_{2}\right) \xi\left(t^{\prime}\right)+\omega_{1}, \\
& \phi_{2}^{\&}=\varepsilon^{2} h_{22}\left(\rho, \theta, \phi_{1}, \phi_{2}\right)+\varepsilon h_{21}\left(\theta, \phi_{1}, \phi_{2}\right) \xi\left(t^{\prime}\right)+\omega_{2}, \\
& \mathrm{~d} \xi\left(t^{\prime}\right)=-\alpha_{0} \xi\left(t^{\prime}\right) \mathrm{d} t+\sigma_{0} \mathrm{~d} W\left(t^{\prime}\right),
\end{aligned}
$$

where

$$
\begin{array}{cc}
\rho_{2}\left(\rho, \theta, \phi_{1}, \phi_{2}\right)=d_{1} \cos \theta \sin \phi_{1}+d_{2} \sin \theta \sin \phi_{2}, s_{2}\left(\rho, \theta, \phi_{1}, \phi_{2}\right)=-d_{1} \sin \theta \sin \phi_{1}+d_{2} \cos \theta \sin \phi_{2}, \\
h_{12}\left(\rho, \theta, \phi_{1}, \phi_{2}\right)=d_{1} \cos ^{-1} \theta \cos \phi_{1}, & h_{22}\left(\rho, \theta, \phi_{1}, \phi_{2}\right)=d_{2} \sin ^{-1} \theta \cos \phi_{2}, \\
\rho_{1}\left(\theta, \phi_{1}, \phi_{2}\right)=d_{3} \cos \theta \sin \phi_{1}+d_{4} \sin \theta \sin \phi_{2}, & s_{1}\left(\theta, \phi_{1}, \phi_{2}\right)=-d_{3} \sin \theta \sin \phi_{1}+d_{4} \cos \theta \sin \phi_{2}, \\
h_{11}\left(\theta, \phi_{1}, \phi_{2}\right)=d_{3} \cos ^{-1} \theta \cos \phi_{1}, & h_{21}\left(\theta, \phi_{1}, \phi_{2}\right)=d_{4} \sin ^{-1} \theta \cos \phi_{2}, \\
d_{1}=-2 \beta \cos \theta \sin \phi_{1}+\gamma c_{1}^{\alpha} e^{-\rho}\left(b_{11} D_{t^{\prime}}^{\alpha}\left(e^{\rho} \cos \theta \cos \phi_{1}\right)+b_{12} D_{t^{\prime}}^{\alpha}\left(e^{\rho} \sin \theta \cos \phi_{2}\right)\right), \\
d_{2}=-2 \beta \sin \theta \sin \phi_{2}+\gamma c_{1}^{\alpha} e^{-\rho}\left(b_{21} D_{t^{\prime}}^{\alpha}\left(e^{\rho} \cos \theta \cos \phi_{1}\right)+b_{22} D_{t^{\prime}}^{\alpha}\left(e^{\rho} \sin \theta \cos \phi_{2}\right)\right), \\
d_{3}=k_{11} \cos \theta \cos \phi_{1}+k_{12} \sin \theta \cos \phi_{2}, & d_{4}=k_{21} \cos \theta \cos \phi_{1}+k_{22} \sin \theta \cos \phi_{2} .
\end{array}
$$

According to Eq. (26), one may let

$$
\phi_{1}=\omega_{1} t^{\prime}+\varphi_{1}, \quad \phi_{2}=\omega_{2} t^{\prime}+\varphi_{2},
$$

where

$$
\begin{aligned}
& \phi \&=\varepsilon^{2} h_{12}\left(\rho, \theta, \phi_{1}, \phi_{2}\right)+\varepsilon h_{11}\left(\theta, \phi_{1}, \phi_{2}\right) \xi\left(t^{\prime}\right), \\
& \phi_{2}^{\&}=\varepsilon^{2} h_{22}\left(\rho, \theta, \phi_{1}, \phi_{2}\right)+\varepsilon h_{21}\left(\theta, \phi_{1}, \phi_{2}\right) \xi\left(t^{\prime}\right) .
\end{aligned}
$$

Hence, we have

$$
\begin{aligned}
& D_{t^{\prime}}^{\alpha}\left(e^{\rho} \cos \theta \cos \phi_{1}\right)=D_{t^{\prime}}^{\alpha}\left(e^{\rho} \cos \theta \cos \varphi_{1} \cos \omega_{1} t^{\prime}\right)-D_{t^{\prime}}^{\alpha}\left(e^{\rho} \cos \theta \sin \varphi_{1} \sin \omega_{1} t^{\prime}\right), \\
& D_{t^{\prime}}^{\alpha}\left(e^{\rho} \sin \theta \cos \phi_{2}\right)=D_{t^{\prime}}^{\alpha}\left(e^{\rho} \sin \theta \cos \varphi_{2} \cos \omega_{2} t^{\prime}\right)-D_{t^{\prime}}^{\alpha}\left(e^{\rho} \sin \theta \sin \varphi_{2} \sin \omega_{2} t^{\prime}\right) .
\end{aligned}
$$


From Eqs. (26-27), one can easily find that $\rho, \theta, \varphi_{1}$, and $\varphi_{2}$ are slow variables. Thus in Eq. (28), they can be considered as constants [38, 39]. Then Eq. (28) is written as

$$
\begin{aligned}
& D_{t^{\prime}}^{\alpha}\left(e^{\rho} \cos \theta \cos \phi_{1}\right)=e^{\rho} \cos \theta \cos \varphi_{1} D_{t^{\prime}}^{\alpha}\left(\cos \omega_{1} t^{\prime}\right)-e^{\rho} \cos \theta \sin \varphi_{1} D_{t^{\prime}}^{\alpha}\left(\sin \omega_{1} t^{\prime}\right), \\
& D_{t^{\prime}}^{\alpha}\left(e^{\rho} \sin \theta \cos \phi_{2}\right)=e^{\rho} \sin \theta \cos \varphi_{2} D_{t^{\prime}}^{\alpha}\left(\cos \omega_{2} t^{\prime}\right)-e^{\rho} \sin \theta \sin \varphi_{2} D_{t^{\prime}}^{\alpha}\left(\sin \omega_{2} t^{\prime}\right) .
\end{aligned}
$$

Applying the fractional derivative formula [40-42], the following is obtained

$$
\begin{aligned}
& D_{t^{\prime}}^{\alpha}\left(\cos \omega t^{\prime}\right)=\omega^{\alpha} \cos \left(\omega t^{\prime}+\frac{\alpha \pi}{2}\right)+\frac{\sin \alpha \pi}{\pi} \int_{0}^{\infty} \frac{v^{1+\alpha} e^{-v t^{\prime}}}{v^{2}+\omega^{2}} d v \\
& D_{t^{\prime}}^{\alpha}\left(\sin \omega t^{\prime}\right)=\omega^{\alpha} \sin \left(\omega t^{\prime}+\frac{\alpha \pi}{2}\right)-\frac{\omega \sin \alpha \pi}{\pi} \int_{0}^{\infty} \frac{v^{\alpha} e^{-v t^{\prime}}}{v^{2}+\omega^{2}} d v
\end{aligned}
$$

Based on the results of Rossikhin [40, 41], as $t^{\prime} \rightarrow \infty$, Eq. (30) becomes

$$
\begin{aligned}
& D_{t^{\prime}}^{\alpha}\left(\cos \omega t^{\prime}\right)=\omega^{\alpha} \cos \left(\omega t^{\prime}+\frac{\alpha \pi}{2}\right), \\
& D_{t^{\prime}}^{\alpha}\left(\sin \omega t^{\prime}\right)=\omega^{\alpha} \sin \left(\omega t^{\prime}+\frac{\alpha \pi}{2}\right) .
\end{aligned}
$$

Substituting Eqs. $(29,31)$ into Eq. (26), we can get the follows

$$
\begin{aligned}
& d_{1}=-2 \beta \cos \theta \sin \phi_{1}+\gamma c_{1}^{\alpha}\left(b_{11} \omega_{1}^{\alpha} \cos \theta \cos \left(\phi_{1}+\alpha \pi / 2\right)+b_{12} \omega_{2}^{\alpha} \sin \theta \cos \left(\phi_{2}+\alpha \pi / 2\right)\right), \\
& d_{2}=-2 \beta \sin \theta \sin \phi_{2}+\gamma c_{1}^{\alpha}\left(b_{21} \omega_{1}^{\alpha} \cos \theta \cos \left(\phi_{1}+\alpha \pi / 2\right)+b_{22} \omega_{2}^{\alpha} \sin \theta \cos \left(\phi_{2}+\alpha \pi / 2\right)\right) .
\end{aligned}
$$

\section{Moment Lyapunov exponent}

Based on the fact of Eqs. $(26,32)$, one is easy to find that the processes $\left(\theta, \phi_{1}, \phi_{2}, \xi\right)$ are independent of the variable $\rho$. Therefore, the processes $\left(\theta, \phi_{1}, \phi_{2}, \xi\right)$ alone form a diffusive Markov process with the following generator:

$$
L(p)=L_{0}(p)+\varepsilon L_{1}(p)+\varepsilon^{2} L_{2}(p),
$$

where

$$
\begin{gathered}
L_{0}(p)=-\alpha_{0} \xi \frac{\partial}{\partial \xi}+\frac{1}{2} \sigma_{0}^{2} \frac{\partial^{2}}{\partial \xi^{2}}+\sum_{i=1}^{2} \omega_{i} \frac{\partial}{\partial \phi_{i}}:=\mathrm{L}_{0}, \\
L_{1}(p)=\xi s_{1}\left(\theta, \phi_{1}, \phi_{2}\right) \frac{\partial}{\partial \theta}+\xi \sum_{i=1}^{2} h_{i 1}\left(\theta, \phi_{1}, \phi_{2}\right) \frac{\partial}{\partial \phi_{i}}+p \xi \rho_{1}\left(\theta, \phi_{1}, \phi_{2}\right):=\mathrm{L}_{1}+p \xi \rho_{1}, \\
L_{2}(p)=s_{2}\left(\theta, \phi_{1}, \phi_{2}\right) \frac{\partial}{\partial \theta}+\sum_{i=1}^{2} h_{i 2}\left(\theta, \phi_{1}, \phi_{2}\right) \frac{\partial}{\partial \phi_{i}}+p \rho_{2}\left(\theta, \phi_{1}, \phi_{2}\right):=\mathrm{L}_{2}+p \rho_{2} .
\end{gathered}
$$

The moment Lyapunov exponent $\Lambda(p)$ of system (26) is the principal simple eigenvalue of the operator $L(p)[16,43]$. i.e., 


$$
L(p) \psi(p)=\Lambda(p) \psi(p)
$$

\subsection{Asymptotic analysis}

According to Khasminskii and Moshchuk [43], both the moment Lyapunov exponent $\Lambda(p)$ and the eigenfunction $\psi(p)$ can be expanded in the power series of $\varepsilon$, respectively, i.e.,

$$
\begin{aligned}
& \Lambda(p)=\Lambda_{0}(p)+\varepsilon \Lambda_{1}(p)+\varepsilon^{2} \Lambda_{2}(p)+\mathrm{L}+\varepsilon^{n} \Lambda_{n}(p)+\mathrm{L}, \\
& \psi(p)=\psi_{0}(p)+\varepsilon \psi_{1}(p)+\varepsilon^{2} \psi_{2}(p)+\mathrm{L}+\varepsilon^{n} \psi_{n}(p)+\mathrm{L} .
\end{aligned}
$$

Substituting Eq. (35) into Eq. (34), the following recursive equations are obtained:

$$
\begin{aligned}
& \varepsilon^{0}:\left(L_{0}(p)-\Lambda_{0}(p)\right) \psi_{0}(p)=0, \\
& \varepsilon^{1}:\left(L_{0}(p)-\Lambda_{0}(p)\right) \psi_{1}(p)=\left(\Lambda_{1}(p)-L_{1}(p)\right) \psi_{0}(p), \\
& \varepsilon^{2}:\left(L_{0}(p)-\Lambda_{0}(p)\right) \psi_{2}(p)=\left(\Lambda_{1}(p)-L_{1}(p)\right) \psi_{1}(p)+\left(\Lambda_{2}(p)-L_{2}(p)\right) \psi_{0}(p), \\
& \quad \mathrm{M}
\end{aligned}
$$

\subsubsection{Zeroth-order perturbation}

From the definition of $\Lambda(p)$, we know that $\Lambda_{0}(p)=0$. Thus, Eq. (36) can be reduced to

$$
\left[-\alpha_{0} \xi \frac{\partial}{\partial \xi}+\frac{\sigma_{0}^{2}}{2} \frac{\partial^{2}}{\partial \xi^{2}}+\omega_{1} \frac{\partial}{\partial \phi_{1}}+\omega_{2} \frac{\partial}{\partial \phi_{2}}\right] \psi_{0}\left(\theta, \phi_{1}, \phi_{2}, \xi\right)=0 .
$$

Using the method of separation of variables and letting

$$
\psi_{0}\left(\theta, \phi_{1}, \phi_{2}, \xi\right)=\Theta(\vartheta) \Phi_{1}\left(\phi_{1}\right) \Phi_{2}\left(\phi_{2}\right) Z(\xi),
$$

Eq. (39) becomes

$$
\frac{\Phi_{1}^{\&}}{\Phi_{1}}=c_{1}, \frac{\Phi_{2}^{\&}}{\Phi_{21}}=c_{2}, \quad-\alpha_{0} \xi \frac{Z^{\&}}{Z}+\frac{\sigma_{0}^{2}}{2} \frac{\&}{Z}=-\left(c_{1} \omega_{1}+c_{2} \omega_{2}\right) .
$$

One can easily obtain that $\Phi_{1}\left(\phi_{1}\right)=C_{1} e^{c_{1} \phi_{1}}$ and $\Phi_{2}\left(\phi_{2}\right)=C_{2} e^{c_{2} \phi_{2}}$. Applying the periodic boundary conditions

$$
\psi_{0}\left(\theta, \phi_{1}+2 \pi, \phi_{2}, \xi\right)=\psi_{0}\left(\theta, \phi_{1}, \phi_{2}+2 \pi, \xi\right)=\psi_{0}\left(\theta, \phi_{1}, \phi_{2}, \xi\right),
$$

we have the constants $c_{1}=c_{2}=0$. Hence, we can get $\Phi_{1}\left(\phi_{1}\right)=C_{1}, \Phi_{2}\left(\phi_{2}\right)=C_{2}$, and

$$
-\alpha_{0} \xi \frac{Z^{\&}}{Z}+\frac{\sigma_{0}^{2}}{2} \frac{z^{K}}{Z}=0
$$

The solution to Eq. (41) is $\mathrm{Z}(\xi)=C_{3}+C_{4} \operatorname{erf}\left(j \sqrt{\alpha_{0}} \xi / \sigma_{0}\right)$, where $\operatorname{erf}(\cdot)$ is the error function and $j$ denotes the imaginary unit. Since $Z(\xi)$ is a bounded function as 
$\xi \rightarrow \pm \infty$, it is required that $C_{4}=0$. i.e., $\mathrm{Z}(\xi)=C_{3}$. Therefore, we obtain that

$$
\psi_{0}\left(\theta, \phi_{1}, \phi_{2}, \xi\right)=\psi_{0}(\theta)
$$

where $\psi_{0}(\theta)$ is an undetermined function.

The adjoint equation of Eq. (39) is

$$
\mathrm{L}_{0}^{*} \psi_{0}^{*}\left(\theta, \phi_{1}, \phi_{2}, \xi\right)=0 .
$$

Utilizing a similar method to solve Eq. (39), one easily gets that

$$
\psi_{0}^{*}\left(\theta, \phi_{1}, \phi_{2}, \xi\right)=\frac{P_{s}(\xi) \mathrm{F}(\theta)}{4 \pi^{2}},
$$

where $\mathrm{F}(\theta)$ is an arbitrary function and $P_{s}(\xi)$ is the stationary probability density of the process $\xi\left(t^{\prime}\right)$.

\subsubsection{First-order perturbation}

Based on the results of section 5.1, Eq. (7) becomes

$$
\mathrm{L}_{0} \psi_{1}=-s_{1}\left(\theta, \varphi_{1}, \varphi_{2}\right) \xi \frac{d \psi_{0}}{d \theta}+\left[\Lambda_{1}(p)-p \rho_{1}\left(\theta, \varphi_{1}, \varphi_{2}\right) \xi\right] \psi_{0} .
$$

Using the solvability condition to Eq. (44), we have

$$
\Lambda_{1}(p)=\left\langle\left(p \rho_{1} \psi_{0}+s_{1} \frac{d \psi_{0}}{d \theta}\right) \xi, \psi_{0}^{*}\right\rangle=\frac{1}{4 \pi^{2}}\left\langle R_{1}\left(\theta, \phi_{1}, \phi_{2} ; p\right) \xi, P_{s}(\xi) \mathrm{F}(\theta)\right\rangle=0,
$$

where

$$
\begin{gathered}
R_{1}\left(\theta, \phi_{1}, \phi_{2} ; p\right)=p \rho_{1}\left(\theta, \phi_{1}, \phi_{2}\right) \psi_{0}(\theta)+s_{1}\left(\theta, \phi_{1}, \phi_{2}\right) \frac{d \psi_{0}(\theta)}{d \theta}, \\
\langle(\cdot),(\cdot)\rangle=\int_{0}^{\pi / 2} \int_{M} \int_{0}^{2 \pi} \int_{0}^{2 \pi}(\cdot)(\cdot) \mathrm{d} \phi_{1} \mathrm{~d} \phi_{2} \mathrm{~d} \xi \mathrm{d} \theta .
\end{gathered}
$$

Therefore, Eq. (44) is reduced to

$$
\mathrm{L}_{0} \psi_{1}=-\xi R_{1}\left(\theta, \varphi_{1}, \varphi_{2} ; p\right)
$$

According to Refs.[18, 20, 23] ,the solution to Eq. (46) is obtained that

$$
\psi_{1}\left(\theta, \phi_{1}, \phi_{2}, \xi ; p\right)=\int_{0}^{+\infty} K(\xi, \tau) R_{1}\left(\theta, \phi_{1}+\omega_{1} \tau, \phi_{2}+\omega_{2} \tau ; p\right) \mathrm{d} \tau,
$$

where

$$
K(\xi, \tau)=\int_{M} \eta P(\eta, \tau ; \xi, 0) \mathrm{d} \eta
$$

$P(\eta, \tau ; \xi, 0)$ is the transient density.

\subsubsection{Second-order perturbation}


Applying the above results in sections 5.1 and 5.2, Eq. (38) is reduced as follows

$$
\begin{aligned}
\mathrm{L}_{0} \psi_{2}= & \left(\Lambda_{2}(p)-p \rho_{2}\left(\theta, \phi_{1}, \phi_{2}\right)\right) \psi_{0}(\theta)-s_{2}\left(\theta, \phi_{1}, \phi_{2}\right) \frac{d \psi_{0}(\theta)}{d \theta} \\
& -L^{1} \psi_{1}\left(\theta, \phi_{1}, \phi_{2}, \xi ; p\right) .
\end{aligned}
$$

The solvability condition to Eq. (48) is

$$
\begin{aligned}
& \left\langle R_{2}\left(\theta, \phi_{1}, \phi_{2}, \xi ; p\right), \psi_{0}^{*}\right\rangle \\
& =\int_{0}^{\pi / 2} \mathrm{~F}(\theta) \int_{M} \int_{0}^{2 \pi} \int_{0}^{2 \pi} \frac{P_{s}(\xi)}{4 \pi^{2}} R_{2}\left(\theta, \phi_{1}, \phi_{2}, \xi ; p\right) \mathrm{d} \phi_{1} \mathrm{~d} \phi_{2} \mathrm{~d} \xi \mathrm{d} \theta \\
& =0,
\end{aligned}
$$

where

$$
\begin{aligned}
& R_{2}\left(\theta, \phi_{1}, \phi_{2}, \xi ; p\right)=\left(\Lambda_{2}(p)-p \rho_{2}\left(\theta, \phi_{1}, \phi_{2}\right)\right) \psi_{0}(\theta)-s_{2}\left(\theta, \phi_{1}, \phi_{2}\right) \frac{d \psi_{0}(\theta)}{d \theta} \\
& \quad-\xi\left(p \rho_{1}\left(\theta, \phi_{1}, \phi_{2}\right)+s_{1}\left(\theta, \phi_{1}, \phi_{2}\right) \frac{\partial}{\partial \vartheta}+\sum_{i=1}^{2} h_{i 1}\left(\theta, \phi_{1}, \phi_{2}\right) \frac{\partial}{\partial \phi_{i}}\right) \psi_{1}\left(\theta, \phi_{1}, \phi_{2}, \xi ; p\right) .
\end{aligned}
$$

Since Eq. (49) always holds for an arbitrary function $\mathrm{F}(\theta)$, it leads to

$$
\int_{M} \int_{0}^{2 \pi} \int_{0}^{2 \pi} \frac{P_{s}(\xi)}{4 \pi^{2}} R_{2}\left(\theta, \phi_{1}, \phi_{2}, \xi ; p\right) \mathrm{d} \phi_{1} \mathrm{~d} \phi_{2} \mathrm{~d} \xi=0
$$

Making use of the correlation function of the process $\xi$ and the power spectral density given, respectively, by

$$
\mathrm{R}(\tau)=\int_{M} \xi P_{s}(\xi) K(\xi, \tau) \mathrm{d} \xi, \quad S(\omega)=2 \int_{0}^{\infty} \mathrm{R}(\tau) \cos \omega \tau \mathrm{d} \tau,
$$

after performing the integration, we obtain

$$
\begin{aligned}
\mathcal{L}(p) \psi_{0}:= & \frac{1}{2} \sigma^{2}(\theta) \frac{d^{2} \psi_{0}}{d \theta^{2}}+(\mu(\theta)+p \hat{\mu}(\theta)) \frac{d \psi_{0}}{d \theta}+\left(p Q(\theta)+\frac{1}{2} p^{2} \hat{Q}(\theta)\right) \psi_{0} \\
& =\Lambda_{2}(p) \psi_{0}
\end{aligned}
$$

where

$$
\begin{gathered}
\sigma^{2}(\theta)=\hat{a} \cos ^{2}(2 \theta)+\hat{b} \cos (2 \theta)+\hat{c}, \\
\mu(\theta)=\sigma^{2}(\theta) \cot (2 \theta)-\Delta \sin (2 \theta), \\
\hat{\mu}(\theta)=\hat{a} \sin (2 \theta) \cos (2 \theta)+\frac{1}{2}(\hat{b}-\hat{d}) \sin (2 \theta), \\
Q(\theta)=\sigma^{2}(\theta)+\Delta \cos (2 \theta)+\hat{e}, \\
\hat{Q}(\theta)=-\hat{a} \cos ^{2}(2 \theta)+\hat{d} \cos (2 \theta)+\hat{f},
\end{gathered}
$$




$$
\begin{gathered}
\hat{a}=\frac{1}{32}\left(\left(k_{12}+k_{21}\right)^{2} S\left(\omega_{+}\right)+\left(k_{12}-k_{21}\right)^{2} S\left(\omega_{-}\right)-\sum_{i=1}^{2} k_{i i}^{2} S\left(2 \omega_{i}\right)\right), \\
\hat{b}=-\frac{1}{16}\left(k_{12}^{2}-k_{21}^{2}\right)\left(S\left(\omega_{+}\right)+S\left(\omega_{-}\right)\right), \\
\hat{c}=\frac{1}{32}\left(\left(k_{12}+k_{21}\right)^{2} S\left(\omega_{-}\right)+\left(k_{12}-k_{21}\right)^{2} S\left(\omega_{+}\right)+\sum_{i=1}^{2} k_{i i}^{2} S\left(2 \omega_{i}\right)\right), \\
\hat{d}=\frac{1}{16}\left(k_{11}^{2} S\left(2 \omega_{1}\right)-k_{22}^{2} S\left(2 \omega_{2}\right)\right), \\
\hat{f}=\frac{1}{32}\left(\left(k_{12}+k_{21}\right)^{2} S\left(\omega_{+}\right)+\left(k_{12}-k_{21}\right)^{2} S\left(\omega_{-}\right)+\sum_{i=1}^{2} k_{i i}^{2} S\left(2 \omega_{i}\right)\right), \\
\Delta_{i}=-\frac{1}{2} \beta-\frac{1}{4} \gamma c_{1}^{\alpha} \sin \frac{\alpha \pi}{2} \omega_{i}^{\alpha} b_{i i}+\frac{1}{16} k_{i i}^{2} S\left(2 \omega_{i}\right), i=1,2 \\
\Delta=\Delta_{1}-\Delta_{2}, \quad \omega_{ \pm}=\omega_{1} \pm \omega_{2} .
\end{gathered}
$$

Since $\varepsilon$ is a small parameter, and $\Lambda_{0}(p)=\Lambda_{1}(p)=0$, the moment Lyapunov exponent can be approximately given as

$$
\Lambda(p) \cong \varepsilon^{2} \Lambda_{2}(p)
$$

Therefore, we can get the approximate analytic expression of moment Lyapunov exponent by solving the eigenvalue of Eq. (51). And the largest Lyapunov exponent can be given by

$$
\lambda=\left.\left.\frac{\mathrm{d}}{\mathrm{d} p} \Lambda(p)\right|_{p=0} \cong \varepsilon^{2} \frac{\mathrm{d}}{\mathrm{d} p} \Lambda_{2}(p)\right|_{p=0} .
$$

\subsection{Solution of the eigenvalue problem}

Based on Refs. [20, 44], the solution $\psi_{0}(\theta)$ to Eq. (51) can be approximated by an orthogonal expansion. According to the nature of the coefficients of the equation, the eigenfunction is expressed as Fourier series. i.e.,

$$
\psi_{0}(\theta)=\sum_{m=0}^{\infty} z_{m} \cos (2 m \theta) .
$$

Substituting Eq. (52) into Eq. (51), we have

$$
\sum_{m=0}^{\infty} c_{m n} z_{m}=\Lambda_{2}(p) z_{n}, \quad n=0,1,2, \mathrm{~L}
$$

where

$$
c_{m n}=\frac{2(1+\operatorname{sgn}(n))}{\pi} \int_{0}^{\pi / 2} \mathscr{L}(p)(\cos (2 m \theta)) \cos (2 n \theta) d \theta, m, n=0,1,2, \mathrm{~L}
$$

Eq. (53) is also presented as 


$$
\left(\mathbf{C}-\Lambda_{2}(p) \mathbf{I}\right) \mathbf{Z}=0
$$

where

$$
\mathbf{C}=\left[\begin{array}{cccc}
c_{00} & c_{01} & c_{02} & \mathrm{~L} \\
c_{10} & c_{11} & c_{12} & \mathrm{~L} \\
c_{20} & c_{21} & c_{22} & \mathrm{~L} \\
\mathrm{M} & \mathrm{M} & \mathrm{M} & \mathrm{O}
\end{array}\right], \quad \mathbf{Z}=\left[z_{0}, z_{1}, z_{2}, \mathrm{~L}, z_{n}, \mathrm{~L}\right]^{T},
$$

and $\mathbf{I}$ is an identity matrix.

In order for Eq. (54) to have a nontrivial solution, the determinant of its corresponding coefficient matrix should be zero. Therefore, the calculation of $\Lambda_{2}(p)$ is transformed into solving the eigenvalue of matrix $\mathbf{C}$. We can obtain a series of approximations by calculating the eigenvalue of a series of submatrices. And the series of approximations will converge to the corresponding true eigenvalues as $n \rightarrow \infty$. However, with the increase of $n$, the amount of calculation will increase drastically. Here, we get the approximations by the truncation of $n$, such as, $\Lambda_{2}(p)=c_{00}$ as $n=0$.

\section{Numerical results and discussions}

In order to verify the accuracy of the results obtained by the perturbation method, Monte Carlo simulation is used to calculate the moment Lyapunov exponent of system (10). Here, the numerical algorithms presented in $[45,46]$ are applied to evaluate the time series and $p$ th moment Lyapunov exponent of system (10). For different cases, the approximate analytical and numerical results of the moment Lyapunov exponent $\Lambda(p)$ are plotted in Fig. 2, and the parameters are given as $b_{11}=1, b_{12}=-1, b_{21}=1, b_{22}=1, k_{11}=1, k_{12}=1, k_{21}=-1, k_{22}=-1$, $\varepsilon=0.1, c_{1}=1.2, \omega_{1}=1, \omega_{2}=3, \beta=0.5, \gamma=0.5, D=0.5, r=0.95, \tau=0.1$. From Fig. 2 ,it can be seen that along with the increase of $n$, the approximate analytical results converge (the moment Lyapunov exponents of the system are almost identical for the cases $n=2$ and $n=3$, which agree well with the numerical results. It shows that the result of the moment Lyapunov exponent $\Lambda(p)$ obtained by perturbation method is correct, and it is enough as $n=3$. Then, based on the approximate analytical results of moment Lyapunov exponent and largest Lyapunov exponent, the stochastic stability of the fractional viscoelastic plate is discussed. 

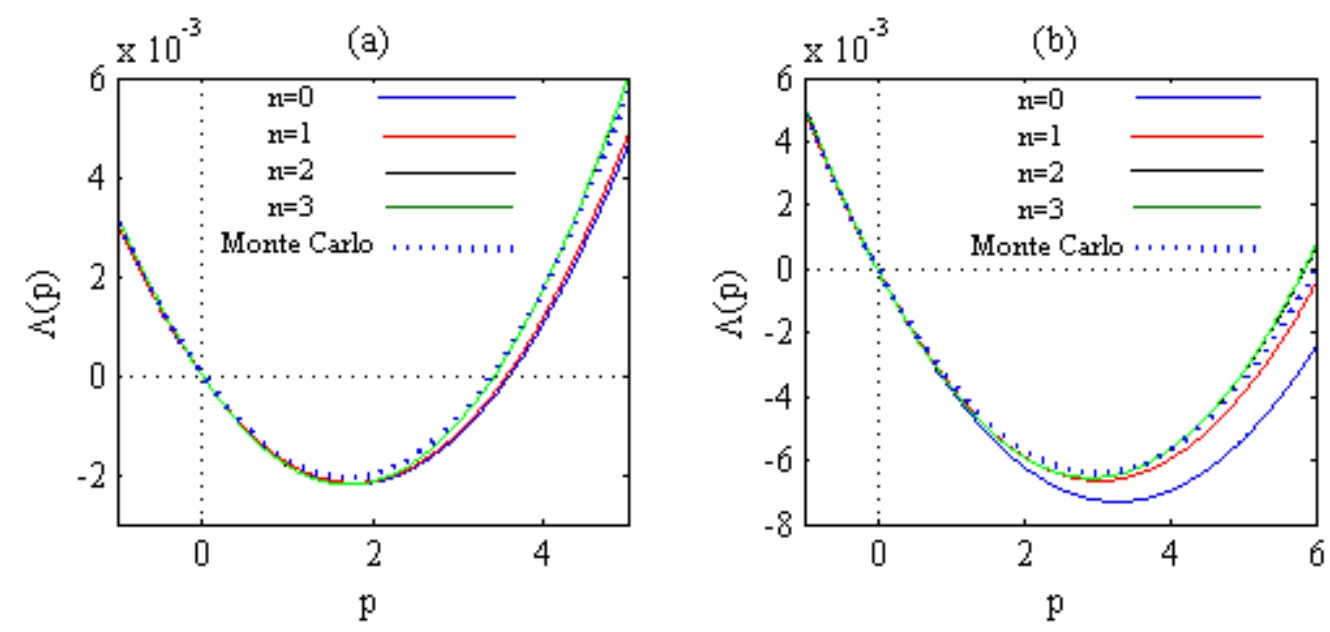

Fig. 2 Comparison of the perturbation results and numerical results of the moment Lyapunov exponent for the case: (a) $\alpha=0.1$; (b) $\alpha=0.5$.

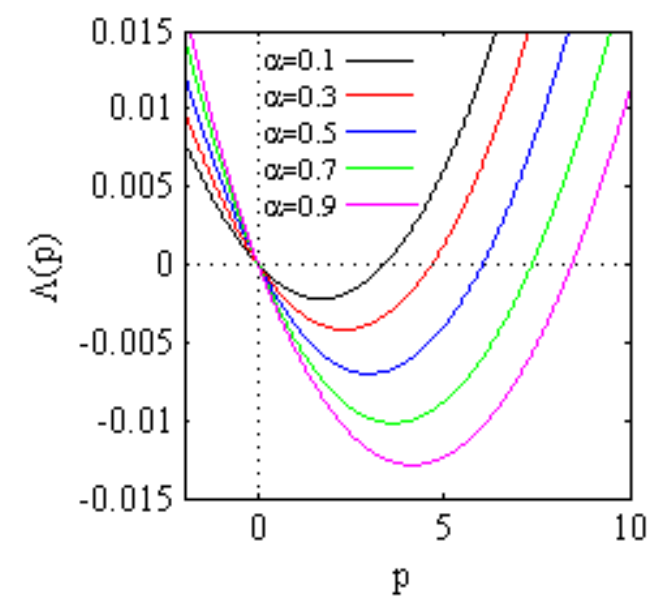

Fig. 3 Effect of the fractional order $\alpha$ on the moment Lyapunov exponent for $D=0.9$.
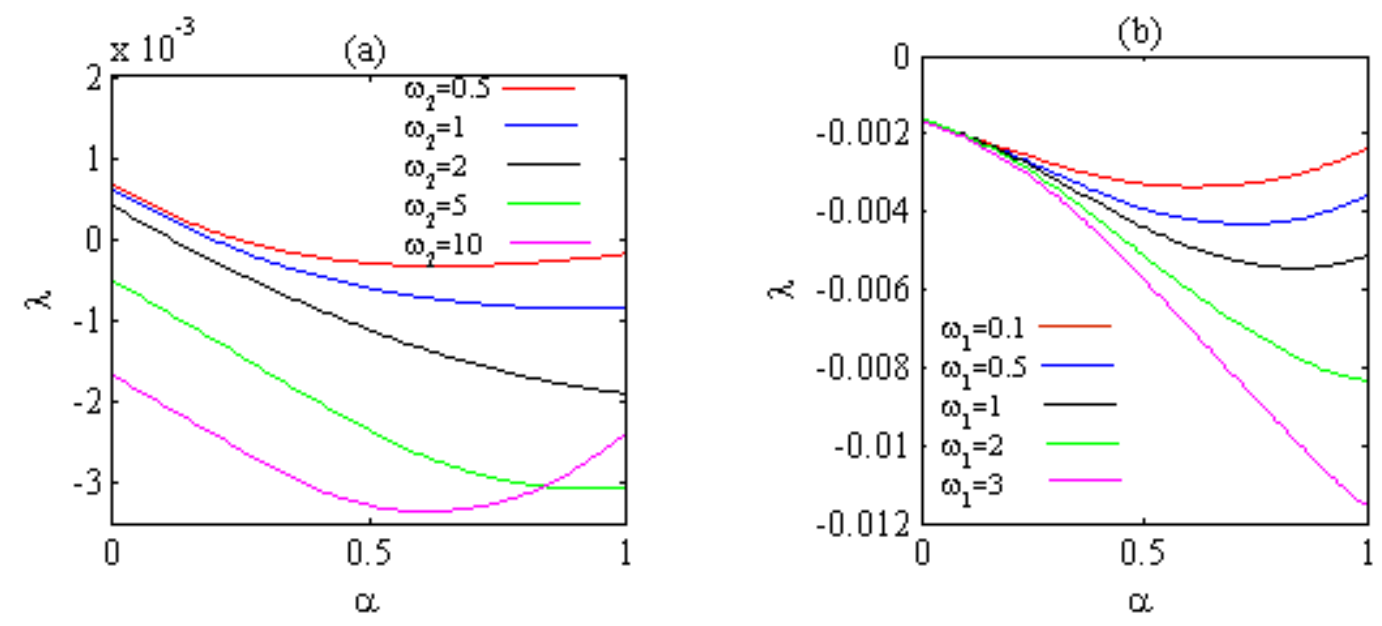

Fig. 4 Largest Lyapunov exponent for $D=0.9$, (a) $\omega_{1}=0.1$; (b) $\omega_{2}=10$.

The effects of fractional order $\alpha$ on the stochastic stability are shown in Figs. 3-6. From Figs. 3-4, it is clear that the moment stability is enhanced and the viscoelastic plate gradually 
becomes stabilized with the increase of the parameter $\alpha$. But the effect of fractional order $\alpha$ on the stochastic stability is not monotonic, which is related to the natural frequencies $\omega_{1}$ and $\omega_{2}$ of the system, as shown in Fig. 4. When the difference between two natural frequencies of the system is large enough or both of them are less than 1, the change curve has a minimum point on the interval $(0,1)$. The effect of the natural frequencies and fractional order on the stochastic stability is plotted in Fig. 5. In the view of Fig. 5, one can observe that with the introduction of fractional order factor, the stability of the system becomes very sensitive to the natural frequencies. According to Eq. (10), one easily finds that the natural frequencies of the system depend on the parameter $a$. Then, the effect of parameter $a$ on the stochastic stability is discussed in Fig. 6. Along with increase of parameter $a$, the almost sure stability of the viscoelastic plate is reduced, and the $\mathrm{D}$ - bifurcation occurs when the parameter $a$ increases to a critical value. From Fig. 6, one can also find that the fractional order $\alpha$ plays an important role in the stochastic stability and D-bifurcation behavior of the system, and the effect of parameter $\alpha$ on the stochastic stability is not monotonic. Therefore, the stochastic stability and D-bifurcation behavior of the system can be controlled by adjusting the fractional order $\alpha$.

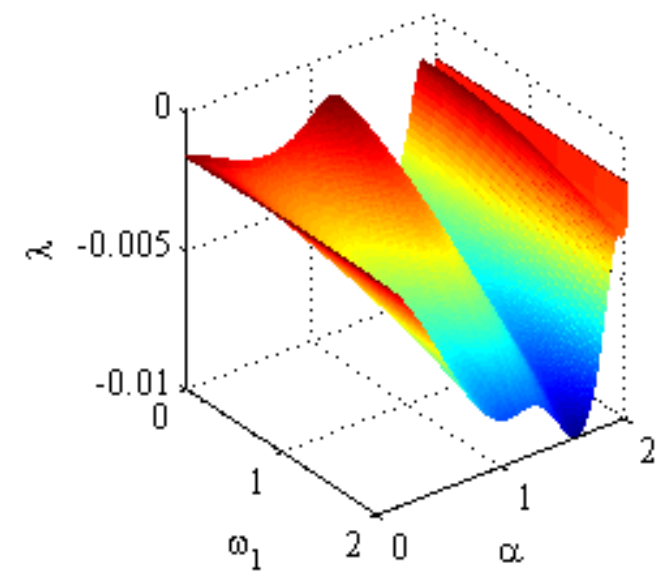

Fig. 5 Largest Lyapunov exponent for $D=0.9, \omega_{2}=10$. 


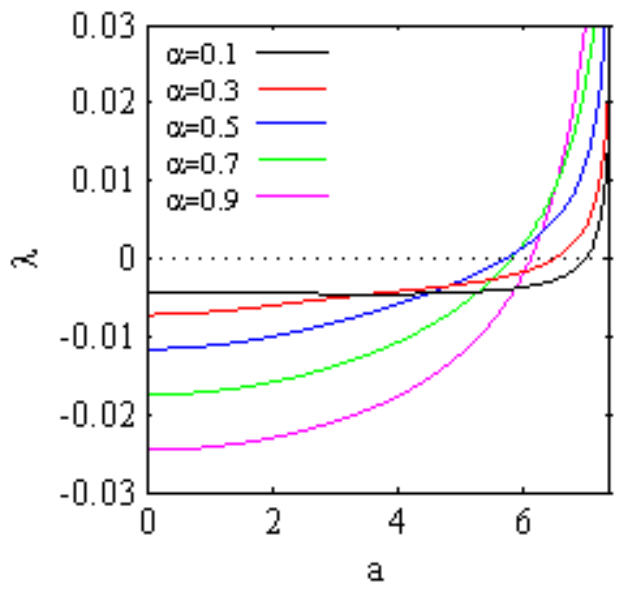

Fig. 6 Largest Lyapunov exponent for $D=0.5, \beta=0.5, \gamma=0.5$.
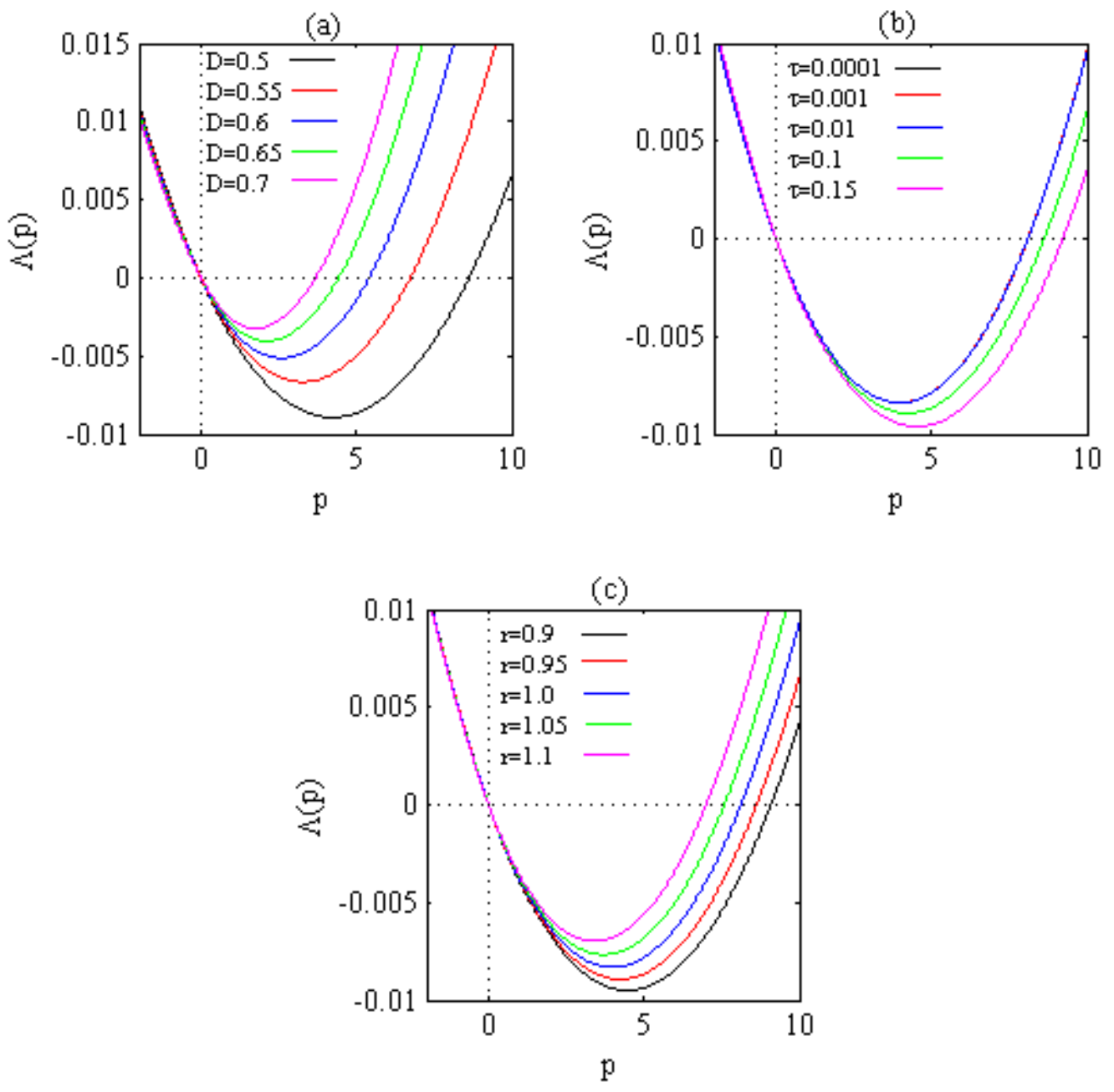

Fig. 7 Effect of noise on the moment Lyapunov exponent for $\alpha=0.1, \beta=0.5, \gamma=0.5, a=1$;
(a) $\tau=0.1, r=0.95$
(b) $D=0.5, r=0.95$;
(c) $D=0.5, \tau=0.1$

The moment Lyapunov exponent and Largest Lyapunov exponent of the system are 
depicted in Figs. 7-8 for different parameters of noise. Based on the fact disclosed in Fig. 7 and Fig. 8, one easily finds that the noise intensity $D$ has a significant effect on the stochastic stability of the viscoelastic plate. Along with the increase of noise intensity $D$, the system will be unstable. And when the noise intensity $D$ is relatively high, the parameter $a$ has a great influence on the stability of the viscoelastic plate. The departure coefficient $r$ can also reduce the stability of the system. However, the effect of the parameter $r$ on the stability of the system is very small as $r \rightarrow 1$, which can be neglected. The noise correlation time $\tau$ will enhance the stability of the system. And in view of Fig. 7(b), it is easily found that the moment Lyapunov exponents of the system are almost identical for the cases $\tau=0.0001, \tau=$ 0.001 , and $\tau=0.01$, which implies the parameter $\tau$ has little effect on the stochastic stability of the system if $\tau$ is very small. Therefore, we should pay more attention to the noise and avoid strong noise as far as possible in practical engineering.

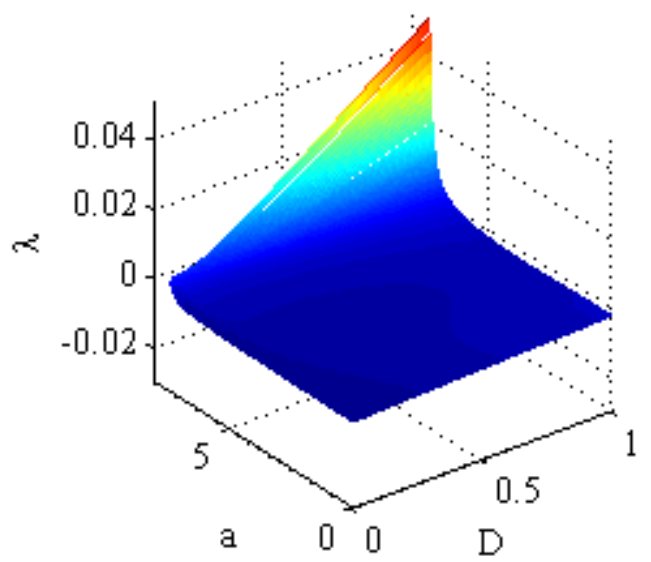

Fig. 8 Largest Lyapunov exponent for $\alpha=0.1, \beta=0.5, \gamma=0.5$.

The effects of parameter $\beta$ on the stochastic stability are shown in Figs. 9-10. It can be found that the stochastic stability of the viscoelastic plate is significantly enhanced with the increase of parameter $\beta$. According to Eqs. (9-10), the value of parameter $\beta$ depends mainly on the damping coefficient $c$. Hence, the damping factor can effectively enhance the stochastic stability of the viscoelastic plate. 


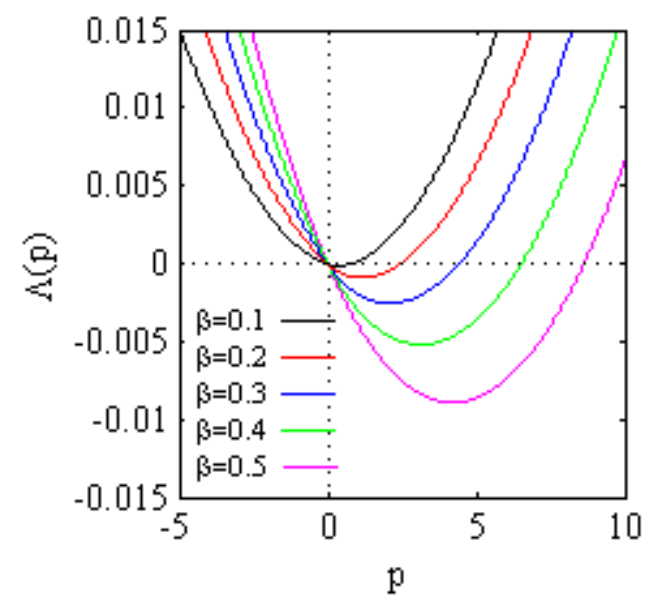

Fig. 9 Effect of damping on the moment Lyapunov exponent for

$$
D=0.5, \alpha=0.1, \gamma=0.5, a=1 .
$$

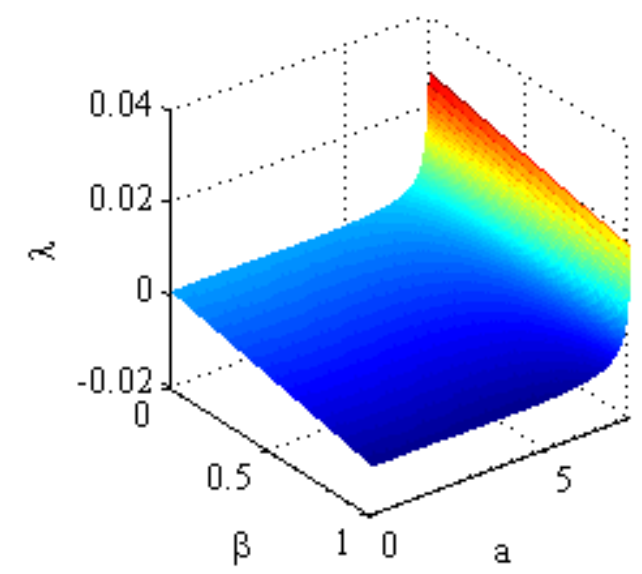

Fig. 10 Largest Lyapunov exponent for $D=0.5, \alpha=0.1, \gamma=0.5$.

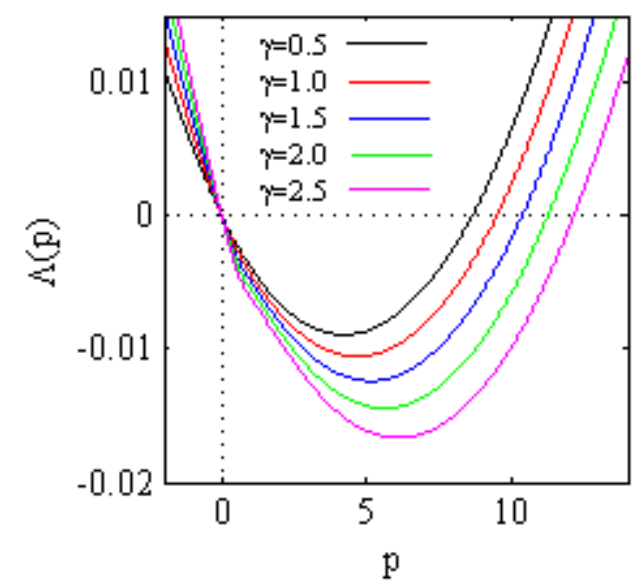

Fig. 11 Effect of viscoelastic on the moment Lyapunov exponent for

$$
D=0.5, \alpha=0.1, \beta=0.5, a=1 .
$$


Figs. 11-12 plot the moment Lyapunov exponent and Largest Lyapunov exponent of the system for different values of viscoelastic coefficient $\gamma$. From Fig. 11, one easily finds that the stochastic stability of the viscoelastic plate can be enhanced with the increase of parameter $\gamma$. But along with the increase of parameter $a$, the effect of viscoelastic coefficient $\gamma$ on the stochastic stability will not be monotonic, as shown in Fig. 12. Therefore, the effect of viscoelastic coefficient $\gamma$ on the stochastic stability of the viscoelastic plate is directly related to the natural frequency of the system.

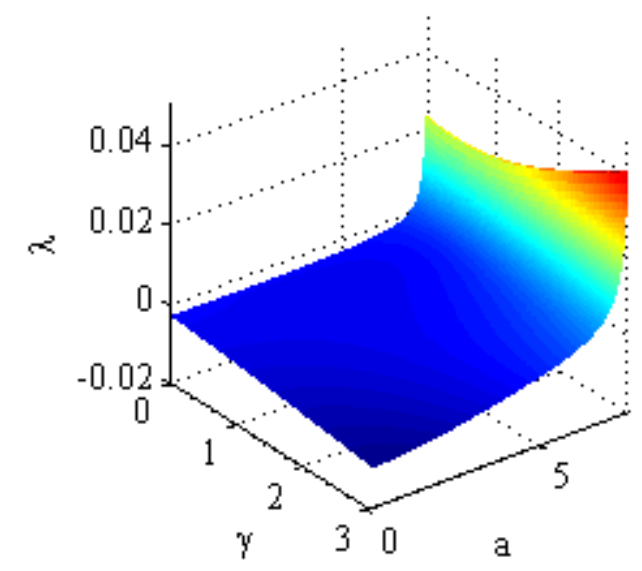

Fig. 12 Largest Lyapunov exponent for $D=0.5, \alpha=0.1, \beta=0.5$.

\section{Conclusion}

Based on the moment Lyapunov exponents, the stochastic dynamics of fractional-order viscoelastic plate under non-Gaussian colored noise excitation is investigated in the present paper. The fractional Kelvin-Voigt constitutive relation is used to describe the material properties of the viscoelastic plate. And applying a path integral approach, the noise is simplified to an Ornstein-Uhlenbeck process. For weak noise excitations, the singular perturbation method is applied to evaluate the moment Lyapunov exponents. And via expanding the eigenfunction as a Fourier series, the approximate analytic solution of the moment Lyapunov exponent is given. Then, the largest Lyapunov exponent is obtained by employing the relationship with the moment Lyapunov exponent. Furthermore, in order to validate the accuracy of the approximate analytical results, the Monte Carlo simulation results are calculated, which agree well with the analytical results. Finally, the effects of the noise and the system parameters on the stochastic stability are discussed, and some results are presented. With the introduction of the fractional Kelvin-Voigt constitutive relation, the natural frequency will affect the stochastic stability of the viscoelastic plate.

Acknowledgments This work in the present paper was supported by the National Natural Science 
Foundation of China (Grant Nos. 11502067 and 11872169), the Fundamental Research Funds for the Central Universities (Grant No. B200202114), and the Six Talent Peaks Project in Jiangsu Province (Grant No. JXQC-002).

\section{Declarations}

Conflict of interest The authors declare that they have no conflict of interest.

\section{Data availability}

All data generated or analysed during this study are included in this published article.

\section{References}

[1] Hryniewicz, Z.: Dynamic analysis of system with deterministic and stochastic viscoelastic dampers. Journal of Sound and Vibration. 278, 1013-1023 (2004)

[2] Zhu, W.Q., Cai, G.Q.: Random vibration of viscoelastic system under broad-band excitations. International Journal of Non-Linear Mechanics. 46, $720-726$ (2011)

[3] Xiong, H., Zhu, W.Q.: A stochastic optimal control strategy for viscoelastic systems with actuator saturation. Probabilistic Engineering Mechanics. 45, 44-51 (2016)

[4] Deng, J.: Dynamic stability of a viscoelastic rotating shaft under parametric random excitation. International Journal of Non-Linear Mechanics. 84, 56-67 (2016)

[5] Liu, Q., Xu, Y., Kurths, J.: Bistability and stochastic jumps in an airfoil system with viscoelastic material property and random fluctuations. Communications in Nonlinear Science and Numerical Simulation. 84, 105184 (2020)

[6] Pavlović, I., Pavlović, R., Kozić, P., Janevski, G.: Almost sure stochastic stability of a viscoelastic double-beam system. Archive of Applied Mechanics. 83, 1591-1605 (2013)

[7] Gu, X.D., Zhu, W.Q.: Optimal bounded control of stochastically excited MDOF nonlinear viscoelastic systems. Nonlinear Dynamics. 89, 125-133 (2017)

[8] Li, X., Gu, J., Xu, W., Ma, F.: Stochastic stability of viscoelastic systems under Gaussian and Poisson white noise excitations. Nonlinear Dynamics. 93, 1579-1588 (2018)

[9] Potapov, V.D.: Stability of viscoelastic plate in supersonic flow under random loading. AIAA Journal. 33, 712-715 (1995)

[10] Ariaratnam, S.T., Abdelrahman, N.M.: Almost-Sure Stochastic Stability of Viscoelastic Plates in Supersonic Flow. AIAA Journal. 39, 465-472 (2001)

[11] Potapov, V.D.: Stability of elastic and viscoelastic plate in gas flow taking into account shear strains under stochastic excitation. Acta Mechanica. 166, 1-12 (2003)

[12] Ling, Q., Jin, X.L., Huang, Z.L.: Response and stability of SDOF viscoelastic system under wideband noise excitations. Journal of the Franklin Institute. 348, 2026-2043 (2011)

[13] Potapov, V.D.: Stability of elastic and viscoelastic systems under stochastic non-Gaussian excitation. Acta Mechanica. 199, 167-179 (2008)

[14] Ariaratnam, S.T., Abdelrahman, N.M.: Stochastic stability of non-gyroscopic viscoelastic systems. International Journal of Solids and Structures. 41, 2685-2709 (2004)

[15] Arnold, L., Kliemann, W.: Large deviations of linear stochastic differential equations, in: Engelbert, H.J., Schmidt, W. (Eds.) Stochastic Differential Systems, pp. 117-151, Springer Berlin Heidelberg, Berlin, Heidelberg, (1987) 
[16] Arnold, L.: A formula connecting sample and moment stability of linear stochastic systems. SIAM Journal on Applied Mathematics. 44, 793-802 (1984)

[17] Kozin, F., Sugimoto, S.: Relations between sample and moment stability for linear stochastic differential equations, in: Proceedings of the conference on stochastic differential equations and applications, pp. 145-162, Academic Press, New York, (1977)

[18] Namachchivaya, N.S., Van Roessel, H.J., Doyle, M.M.: Moment Lyapunov exponent for two coupled oscillators driven by real noise. SIAM Journal on Applied Mathematics. 56, 1400-1423 (1996)

[19] Arnold, L., Doyle, M.M., Namachchivaya, N.S.: Small noise expansion of moment Lyapunov exponents for two-dimensional systems. Dynamics and stability of systems. 12, 187-211 (1997)

[20] Namachchivaya, N.S., Van Roessel, H.J.: Moment Lyapunov exponent and stochastic stability of two coupled oscillators driven by real noise. ASME Journal of Applied Mechanics. 68, 903-914 (2001)

[21] Xie, W.C.: Moment Lyapunov exponents of a two-dimensional system under bounded noise parametric excitation. Journal of Sound and Vibration. 263, 593-616 (2003)

[22] Kozić, P., Janevski, G., Pavlović, R.: Moment Lyapunov exponents and stochastic stability of a double-beam system under compressive axial loading. International Journal of Solids and Structures. 47, 1435-1442 (2010)

[23] Hu, D.L., Huang, Y., Liu, X.B.: Moment Lyapunov exponent and stochastic stability of binary airfoil driven by non-Gaussian colored noise. Nonlinear Dynamics. 70, 1847-1859 (2012)

[24] Hu, D.L., Liu, X.B., Chen, W.: Moment Lyapunov exponent and stochastic stability of binary airfoil under combined harmonic and Gaussian white noise excitation. Nonlinear Dynamics. 89, 539-552 (2017)

[25] Huang, Y., Li, S.H., Liu, X.B.: On the moment Lyapunov exponent of a viscoelastic plate subjected to the excitation of wide band noises Chinese Journal of Theoretical and Applied Mechanics. 43, 551-560 (2011)

[26] Huang, Y., Liu, X.: Stochastic stability of viscoelastic system under non-Gaussian colored noise excitation. Science China Physics, Mechanics and Astronomy. 55, 483-492 (2012)

[27] Deng, J., Zhong, Z., Liu, A.: Stochastic stability of viscoelastic plates under bounded noise excitation. European Journal of Mechanics - A/Solids. 78, 103849 (2019)

[28] Wu, J.C., Liu, X.B.: Moment stability of viscoelastic system influenced by non-Gaussian colored noise. Journal of Sound and Vibration. 502, 116080 (2021)

[29] Li, G.-g., Zhu, Z.-y., Cheng, C.-j.: Dynamical Stability of Viscoelastic Column with Fractional Derivative Constitutive Relation. Applied Mathematics and Mechanics. 22, 294-303 (2001)

[30] Floris, C.: Stochastic stability of a viscoelastic column axially loaded by a white noise force. Mechanics Research Communications. 38, 57-61 (2011)

[31] Deng, J., Xie, W.C., Pandey, M.D.: Stochastic stability of a fractional viscoelastic column under bounded noise excitation. Journal of Sound and Vibration. 333, 1629-1643 (2014)

[32] Bouzat, S., Wio, H.S.: New aspects on current enhancement in Brownian motors driven by non-Gaussian noises. Physica A: Statistical Mechanics and its Applications. 351, 69-78 (2005)

[33] Lu, L., Jia, Y., Ge, M., Xu, Y., Li, A.: Inverse stochastic resonance in Hodgkin-Huxley neural system driven by Gaussian and non-Gaussian colored noises. Nonlinear Dynamics. 100, 877-889 (2020)

[34] Zhang, H., Xu, W., Guo, Q., Han, P., Qiao, Y.: First escape probability and mean first exit time for a time-delayed ecosystem driven by non-Gaussian colored noise. Chaos, Solitons \& Fractals. 135, 109767 (2020) 
[35] Bolotin, V.V., Grishko, A.A., Petrovsky, A.V.: Secondary bifurcations and global instability of an aeroelastic non-linear system in the divergence domain. Journal of Sound \& Vibration. 191, 431-451 (1996)

[36] Fuentes, M.A., Wio, H.S., Toral, R.: Effective Markovian approximation for non-Gaussian noises: a path integral approach. Physica A: Statistical Mechanics and its Applications. 303, 91-104 (2002)

[37] Fuentes, M.A., Toral, R., Wio, H.S.: Enhancement of stochastic resonance: the role of non Gaussian noises. Physica A: Statistical Mechanics and its Applications. 295, 114-122 (2001)

[38] Chen, L.C., Zhu, W.Q.: Stochastic stability of Duffing oscillator with fractional derivative damping under combined harmonic and white noise parametric excitations. Acta Mechanica. 207, 109-120 (2009)

[39] Chen, L.C., Zhu, W.Q.: Stochastic jump and bifurcation of Duffing oscillator with fractional derivative damping under combined harmonic and white noise excitations. International Journal of Non-Linear Mechanics. 46, 1324-1329 (2011)

[40] Rossikhin, Y.A., Shitikova, M.V.: New approach for the analysis of damped vibrations of fractional oscillators. Shock \& Vibration. 16, 365-387 (2009)

[41] Rossikhin, Y.A., Shitikova, M.V.: On fallacies in the decision between the Caputo and RiemannLiouville fractional derivatives for the analysis of the dynamic response of a nonlinear viscoelastic oscillator. Mechanics Research Communications. 45, 22-27 (2012)

[42] Xu, Y., Li, Y., Liu, D.: A method to stochastic dynamical systems with strong nonlinearity and fractional damping. Nonlinear Dynamics. 83, 2311-2321 (2016)

[43] Khasminskii, R., Moshchuk, N.: Moment Lyapunov exponent and stability index for linear conservative system with small random perturbation. SIAM Journal on Applied Mathematics. 58, 245-256 (1998)

[44] Bolotin, V.V.: The dynamic stability of elastic systems. Holden-Day, (1964)

[45] Deng, W.: Numerical algorithm for the time fractional Fokker-Planck equation. Journal of Computational Physics. 227, 1510-1522 (2007)

[46] Xie, W.C., Huang, Q.H.: Simulation of moment Lyapunov exponents for linear homogeneous stochastic systems. ASME Journal of Applied Mechanics. 76, 031001 (2009) 